\title{
Dufour and Soret effect on Double-Diffusive Electrically conducting boundary layer flows in a vertical plate considering internal heat and mass generation
}

\author{
${ }^{1}$ M. Ferdows, ${ }^{2}$ S. M. Chapal, ${ }^{3}$ M. A.A. Hamad, ${ }^{4}$ Raushan Ara Quadir \\ ${ }^{I}$ Department of Mathematics, University of Dhaka, Dhaka-1000, Bangladesh \\ ${ }^{2}$ Department of Mathematics, Jagannath University, Dhaka-1100, Bangladesh \\ ${ }^{3}$ Mathematics Department, Faculty of Science, Assiut University, Assiut 71516, Egypt. \\ ${ }^{4}$ School of Mathematics, Statistics and Computer Science, University of KwaZulu-Natal, Durban, South Africa.
}

\begin{abstract}
The two dimensional steady laminar MHD free convection heat and mass transfer flow over a vertical plate in an incompressible, viscous and electrically conducting fluid is examined considering the wall temperature and concentration distribution proportional to $x^{\lambda}$ utilizing the similarity transformations. The transformed nonlinear boundary layer equations have been obtained by solving the governing equations using numerical technique. Numerical calculations are carried out for different values of dimensionless parameters and an analysis of the results obtained shows that the flow is influenced of magnetic parameter, mixed convection parameter, Lewis, Dufour and Soret number in the fluid. The obtained results are presented graphically and in tabular form.
\end{abstract}

Key-words: Double diffuse, Internal heat generation, Internal mass generation, MHD, Maple.

Nomenclature

C Concentration

$C_{p} \quad$ Specific heat at constant pressure

$C_{s} \quad$ Concentration susceptibility

$D_{f} \quad$ Dufour number

$D_{m} \quad$ Mass diffusivity

$f \quad$ Dimensionless stream fuction

$H_{0} \quad$ Magnetic field intensity

$K \quad$ Darcy permeability

$k_{T} \quad$ Thermal diffusion ratio

Le Lewis number

$M \quad$ Magnetic parameter

$N \quad$ Sustentation parameter

$R a_{x} \quad$ Local Rayleigh number

$P e_{x} \quad$ peclet number

$u, v \quad$ Velocities in the $x$ and $y$ - direction repectively

$T \quad$ Temperature $x, y$ cartesian coordinates normal to the plate and along it, respectively

$\alpha_{m} \quad$ Thermal diffusivity

$\beta_{T} \quad$ Coefficient of thermal expansion

$\beta_{C} \quad$ Coefficient of concentration expansion

$A, B \quad$ Constant

$\phi \quad$ Dimensionless concentration

$\mu \quad$ Viscosity

$\mu_{e} \quad$ Magnetic permeability

$v \quad$ Kinematic viscosity

$\theta \quad$ Dimensionless temperature

$\sigma \quad$ Electrical conductivity

$\rho \quad$ Density

$\psi \quad$ Stream function

E Constant

$q^{\prime \prime \prime} \quad$ Internal heat generation per volume

$\varepsilon \quad$ Mixed convection parameter

\section{Introduction}

It is now well known that most of the fluids used in industry do not hold commonly accepted assumptions of a linear relationship between the stress and the rate of strain and, thus are characterized as nonNewtonian fluids. The flows of such fluids present exciting challenges to engineers, mathematicians, physicists, modelers and numerical simulists. The inadequacies of the classical Navier-Stokes theory to describe rheological complex fluids such as polymer solutions, blood, paints, certain oils, and greases, have led to the development of several theories of non-Newtonian fluids. In this theory, relation connecting shear stress and shear rate is not usually linear; that is, the 'viscosity' of a non- Newtonian fluid is not constant at a given temperature and pressure, but depends on therate of shear or on the previous kinematic history of the fluid [1]. 
The analysis of mixed convection boundary layer flow along a vertical plate embedded in viscous fluids has received considerable theoretical and practical interest. The phenomenon of mixed convection occurs in many technical and industrial problems such as electronic devices cooled by fans, nuclear reactors cooled during an emergency shutdown, a heat exchanger placed in a low-velocity environment, solar collectors and so on. Several authors have studied the problem of mixed convection about different surface geometries. When heat and mass transfer occur simultaneously in a moving fluid, the relations between the fluxes and the driving potentials are of a more intricate nature. It has been observed that an energy flux can be generated not only by temperature gradients but also by concentration gradients. The energy flux caused by a concentration gradient is termed the diffusion-thermo (Dufour) effect. On the other hand, mass fluxes can also be created by temperature gradients and this embodies the thermal-diffusion (Soret) effect. In most of the studies related to heat and mass transfer process, Soret and Dufour effects are neglected on the basis that they are of a smaller order of magnitude than the effects described by Fouriers and Ficks laws. But these effects are considered as second order phenomena and may become significant in areas such as hydrology, petrology, geosciences, etc. The Soret effect, for instance, has been utilized for isotope separation and in mixture between gase with very light molecular weight $(\mathrm{H} 2, \mathrm{He})$ and of medium molecular weight $(\mathrm{N} 2$, air). The Dufour effect was recently found to be of order of considerable magnitude such that it cannot be neglected by Eckeret and Drake [2]. Dursunkaya and Worek [3] studied diffusion-thermo and thermal-diffusion effects in transient and steady natural convection from a vertical surface, whereas Kafoussias and Williams [4] presented the same effects on mixed convective and mass transfer transfer steady laminar boundary layer flow over a vertical flat plate with temperature dependent viscosity. Postelnicu [5] studied numerically the influence of a magnetic field on heat and mass transfer by natural convection from vertical surfaces in porous media considering Soret and Dufour effects. Both free and forced convection boundary layer flows with Soret and Dufour have been addressed by Abreu et al. [6]. Recently, the effect of Soret and Dufour parameters on free convection heat and mass transfers from a vertical surface in a doubly stratified Darcian porous medium has been reported by Lakshmi Narayana and Murthy [7].

The study of non-Newtonian fluid flows has gained much attention from the researchers because of its applications in biology, physiology, technology and industry. In addition, the effects of heat and mass transfer in non-Newtonian fluid also have great importance in engineering applications like the thermal design of industrial equipment dealing with molten plastics, polymeric liquids, foodstuffs, or slurries. Several investigators have extended many of the available convection heat and mass transfer problems to include the non-Newtonian effects. Many of the non-Newtonian fluid models describe the nonlinear relationship between stress and the rate of strain. But the micropolar fluid model introduced by Eringen [8] exhibits some microscopic effects arising from the local structure and micro motion of the fluid elements. Further, they can sustain couple stresses and include classical Newtonian fluid as a special case. The model of micropolar fluid represents fluids consisting of rigid, randomly oriented (or spherical) particles suspended in a viscous medium where the deformation of the particles is ignored. Micropolar fluids have been shown to accurately simulate the flow characteristics of polymeric additives, geomorphological sediments, colloidal suspensions, haematological suspensions, liquid crystals, lubricants etc. The mathematical theory of equations of micropolar fluids and applications of these fluids in the theory of lubrication and porous media is presented by Lukaszewicz [9]. The heat and mass transfer in micropolar fluids is also important in the context of chemical engineering, aerospace engineering and also industrial manufacturing processes. The problem of mixed convection heat and mass transfer in the boundary layer flow along a vertical surface submerged in a micropolar fluid has been studied by a number of investigators. Ahmadi [10] studied the boundary layer flow of a micropolar fluid over a semi-infinite plate. Laminar mixed convection boundary layer flow of a micropolar fluid from an isothermal vertical flat plate has been considered by Jena and Mathur [11]. Gorla [12] presented asymptotic boundary layer solutions in order to study the combined convection from a vertical semi-infinite plate to a micropolar fluid with uniform heat flux. A two-dimensional steady convective flow of thermo-micropolar fluid past a vertical permeable flat plate in the presence of heat generation with uniform surface heat flux has been analyzed numerically by Rahman et al. [13]. Although the Soret and Dufour effects of the medium on the heat and mass transfer in a micropolar fluid is important, very little work has been reported in the literature. Beg et al. [14] analyzed the two dimensional coupled heat and mass transfer of an incompressible micropolar fluid past a moving vertical surface embedded in a Darcy- Forchheimer porous medium in the presence of significant Soret and Dufour effects. A mathematical model for the steady thermal convection heat and mass transfer in a micropolar fluid saturated Darcian porous medium in the presence of significant Dufour and Soret effects and viscous heating is presented by Rawat and Bhargava [15]. Soret and dufour effects on steady MHD free convection flow past a semi-infinite moving vertical plate in a porous medium with viscous dissipation studied by Gnaneswara Reddy and Bhaskar Reddy [16]. Mathematical modeling of transient magnetohydrodynamic couple stress fluid flow in a rotating channel presented by Ghosh et al. [17]. Ramana Murthy et al. [18] considered the finite difference solution for MHD flow of couple stress fluid between two concentric rotating cylinders with porous lining. 
The objective of the present paper is to study Soret and Dufour effects on the mixed convection from a vertical plate embedded in electrically conducting fluid saturated porous medium. The effects of magnetic parameter, mixed convection parameter, sustentation parameter, Lewis, Soret and Dufour numbers are examined and are displayed through graphs.

\section{Mathematical formulation}

We consider a steady two-dimensional laminar flow of a viscous incompressible, electrically conducting fluid past a vertical plate embedded in a porous medium. We assume the flow to be in the $x-$ direction, which is taken along the vertical plate and the $y$-axis to be normal to it. The plate is maintained at a constant temperature $T_{w}$, which is higher than the free stream temperature $T_{\infty}$ of the surrounding fluid and a constant concentration $C_{w}$ which is greater than the constant concentration $C_{\infty}$ of the surrounding fluid. Several assumptions are used throughout the present paper: (a) the fluid and the porous medium are in local thermodynamic equilibrium; (b) the flow is laminar, steady-state and two-dimensional; (c) the porous medium is isotropic and homogeneous; (d) the properties of the fluid and porous medium are constants; (e) the Boussinesq approximation is valid and the boundary-layer approximation is applicable [5].

In-line with these assumptions, the governing equations describing the conservation of mass, momentum, energy and concentration can be written as follows:

$$
\begin{aligned}
& \frac{\partial u}{\partial x}+\frac{\partial v}{\partial y}=0 \\
& u\left(1+\frac{K \sigma \mu_{e}^{2} H_{0}^{2}}{\mu}\right)=\frac{g K}{v}\left[\beta_{T}\left(T-T_{\infty}\right)+\beta_{C}\left(C-C_{\infty}\right)\right] \\
& u \frac{\partial T}{\partial x}+v \frac{\partial T}{\partial y}=\alpha_{m} \frac{\partial^{2} T}{\partial y^{2}}+\frac{D_{m}}{C_{s}} \frac{\kappa_{T}}{C_{p}} \frac{\partial^{2} C}{\partial y^{2}}+q_{h}^{\prime \prime \prime} \\
& u \frac{\partial C}{\partial x}+v \frac{\partial C}{\partial y}=D_{m} \frac{\partial^{2} C}{\partial y^{2}}+\frac{D_{m} \kappa_{T}}{T_{m}} \frac{\partial^{2} C}{\partial y^{2}}+q_{m}^{\prime \prime \prime}
\end{aligned}
$$

where $\alpha_{m}$ and $D_{m}$ are the thermal diffusivity and mass diffusivity, $C_{p}$ and $C_{s}$ are the specific heat at constant pressure and concentration susceptibility, $\kappa_{T}$ is the thermal ratio, $q^{\prime \prime \prime}$ is the internal heat generation per volume, $\sigma, \mu_{e}$ and $H_{0}$ are electrical conductivity, magnetic permeability and magnetic field intensity, respectively.

The appropriate boundary conditions of the problem are

$$
\begin{array}{lr}
v=V_{0}(x), T=T_{w}=T_{\infty}+A x^{\lambda}, C=C_{w}=C_{\infty}+B x^{\lambda} & \text { at } \eta=0 \\
u \rightarrow U_{\infty}, T \rightarrow T_{\infty}, C \rightarrow C_{\infty} & \text { as } \eta \rightarrow \infty
\end{array}
$$

where $U_{\infty}\left(=a x^{m}\right)$ is the uniform velocity and $V_{0}(x)$ is the velocity of suction at the plate.

By introducing the following non-dimensional variables

$$
\begin{aligned}
& \psi=\alpha_{m}\left(P e_{x}\right)^{\frac{1}{2}} f(\eta), \quad \theta(\eta)=\frac{T-T_{\infty}}{T_{w}-T_{\infty}}, \phi(\eta)=\frac{C-C_{\infty}}{C_{w}-C_{\infty}} \\
& \eta=\frac{y}{x}\left(P e_{x}\right)^{\frac{1}{2}}, \quad P e_{x}=\frac{U_{\infty} x}{\alpha_{m}}, \quad U_{\infty}=E x^{m} \\
& q_{h}^{\prime \prime \prime}=\frac{\alpha_{m}\left(T_{w}-T_{\infty}\right)}{x^{2}\left(P e_{x}\right)^{-1}} e^{-\eta}, \quad q_{m}^{\prime \prime \prime}=\frac{\alpha_{m}\left(C_{w}-C_{\infty}\right)}{x^{2}\left(P e_{x}\right)^{-1}} e^{-\eta}
\end{aligned}
$$

where $f(\eta)$ is the dimensionless stream function and $\psi$ is the dimensional stream function defined by $u=\frac{\partial \psi}{\partial y}$ and $v=-\frac{\partial \psi}{\partial x}$, just to satisfy the equation of continuity(1). 
Then introducing the relation (6), into equation (1) we obtain

$$
u=U_{\infty} f^{\prime}(\eta) \text { and } v=-\sqrt{\frac{\alpha_{m} U_{\infty}}{x}}\left[\left(\frac{m+1}{2}\right) f+\left(\frac{m-1}{2}\right) \eta f^{\prime}\right]
$$

Using (6) and (7), equations (2) to (4) become

$$
\begin{aligned}
& (1+M) f^{\prime}=\varepsilon \theta+N \varepsilon \phi \\
& \theta^{\prime \prime}-\lambda f^{\prime} \theta+\left(\frac{m+1}{2}\right) f \theta^{\prime}+D_{f} \phi^{\prime \prime}+c_{h} e^{-\eta}=0 \\
& \frac{1}{L e} \phi^{\prime \prime}-\lambda f^{\prime} \phi+\left(\frac{m+1}{2}\right) f \phi^{\prime}+S_{r} \theta^{\prime \prime}+c_{m} e^{-\eta}=0
\end{aligned}
$$

where $M=\frac{K \sigma \mu_{e}{ }^{2} H_{0}{ }^{2}}{\mu}$ is the magnetic parameter, $\varepsilon=\frac{R a_{x}}{P e_{x}} \quad$ is the mixed convection parameter, $N=\frac{\beta_{C}\left(C_{w}-C_{\infty}\right)}{\beta_{T}\left(T_{w}-T_{\infty}\right)}$ is the sustentation parameter,$L e=\frac{\alpha_{m}}{D_{m}} \quad$ is the Lewis number, $D_{f}=\frac{D_{m} \kappa_{T}\left(T_{w}-T_{\infty}\right)}{C_{s} C_{p} \alpha_{m}\left(C_{w}-C_{\infty}\right)}$ is the Dufour number and $S_{r}=\frac{D_{m} \kappa_{T}\left(T_{w}-T_{\infty}\right)}{T_{m} \alpha_{m}\left(C_{w}-C_{\infty}\right)}$ is the Soret number .

The corresponding boundary conditions (5) become

$$
f=f_{w}, \theta=1, \phi=1 \quad \text { at } \eta=0
$$

$f^{\prime}=1, \theta=0, \phi=0 \quad$ as $\eta \rightarrow \infty$

where $f_{w}=-\frac{2 V_{0}}{m+1} \sqrt{\frac{x}{\alpha_{m} U_{\infty}}}$ is the dimensionless suction velocity and primes denote partial differentiation with respect to the variable $\eta$.

\section{Results and Discussions:}

In order to have a physical point of view of the problem, numerical calculations were carried out for different values of suction parameter $\left(f_{w}\right)$, magnetic parameter $(M)$, mixed convection parameter $(\varepsilon)$, sustentation parameter $(N)$, Lewis number $(L e)$, Dufour number $D_{f}$ and Soret number $(S r)$.

The numerical results are illustrated in Figure 1 to Figure 24 to show the influences of different parameters on the boundary layer flow. Figures 1-4 demonstrate the typical profiles for the displacement, velocity, temperature and concentration functions with and without IHG for different values of Lewis number $(L e)$ when $M=1, N=1, m=1, D_{f}=0.15, S_{r}=0.4, \varepsilon=0.4, \lambda=1 / 4$, respectively. These results show that the distance, velocity and concentration profiles decreases with increasing Lewis number $L e$, whereas the temperature profiles increases with increasing Lewis number $L e$.

Effects of magnetic parameter $M$ on the distance $f(\eta)$, velocity $f^{\prime}(\eta)$, temperature $\theta(\eta)$ and concentration $\phi(\eta)$ profiles are shown with and without IHG through figures 5-8 for various values of magnetic parameter $M$ when $L e=5, N=1, m=1, D_{f}=0.15, S_{r}=0.4, \varepsilon=0.4, \lambda=1 / 4$. These results show that the distance and velocity profiles are decreases with increasing magnetic parameter $M$, while the reverse trend is seen for temperature and concentration profiles. 
The influence of mixed convection parameter $\varepsilon$ on the distance, velocity, temperature and concentration profiles with and without IHG has been illustrated through figures 9-12. It is observed that, while all other participating parameters are held constant and $\varepsilon$ is increased, it is seen that the distance and velocity profiles increases with increasing mixed convection parameter $\varepsilon$, whereas the temperature and concentration profiles decreases with increasing mixed convection parameter $\varepsilon$.

Figures 13-16 are the graphical representations of the distance $f(\eta)$, velocity $f^{\prime}(\eta)$, temperature $\theta(\eta)$ and concentration $\phi(\eta)$ profiles with and without IHG for different values of $\lambda$ when $L e=5, N=1, m=1, D_{f}=0.15, S_{r}=0.4, M=1, \varepsilon=0.4$. It is found from these figures that the velocity, temperature and concentration profiles are decreases with increasing exponent parameter $\lambda$, while the reverse trend is seen for the distance profiles.

The effects of $m$ on the distance, velocity, temperature and concentration profiles are illustrated with and without IHG through figures 17-20 for various values of $m$. It is seen from these figures that the distance, velocity, temperature and concentration profiles are deceases with increasing $m$.

Figures 21-24 demonstrate the typical profiles for the displacement, velocity, temperature and concentration profiles with and without IHG for different values of Dufour and Soret number $\left(D_{f}, S_{r}\right)$ when $L e=5, N=1, m=1, \varepsilon=0.4, M=1, \lambda=1 / 4$. It is observed from these figures that the distance, velocity and concentration profiles increases with decreasing Dufour and Soret number $\left(D_{f}, S_{r}\right)$, whereas the temperature profiles decreases with decreasing Dufour and Soret number $\left(D_{f}, S_{r}\right)$.

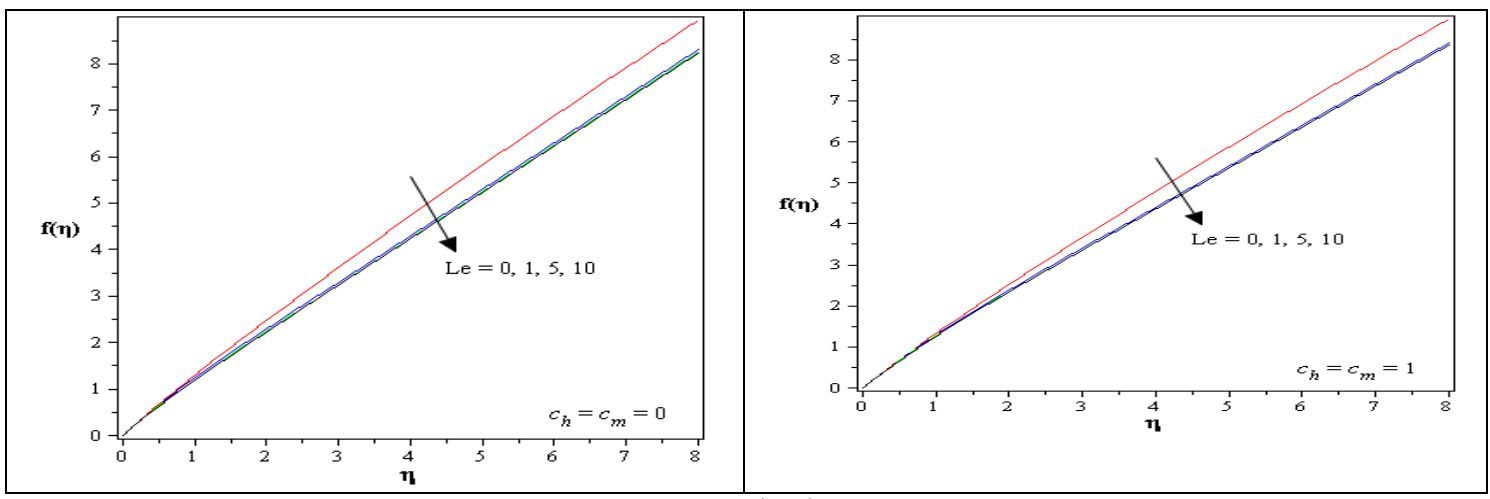

Fig. 1: Effects of Lewis number $(L e)$ on distance profiles when

$M=1, N=1, m=1, D_{f}=0.15, S_{r}=0.4, \varepsilon=0.4, \lambda=1 / 4$

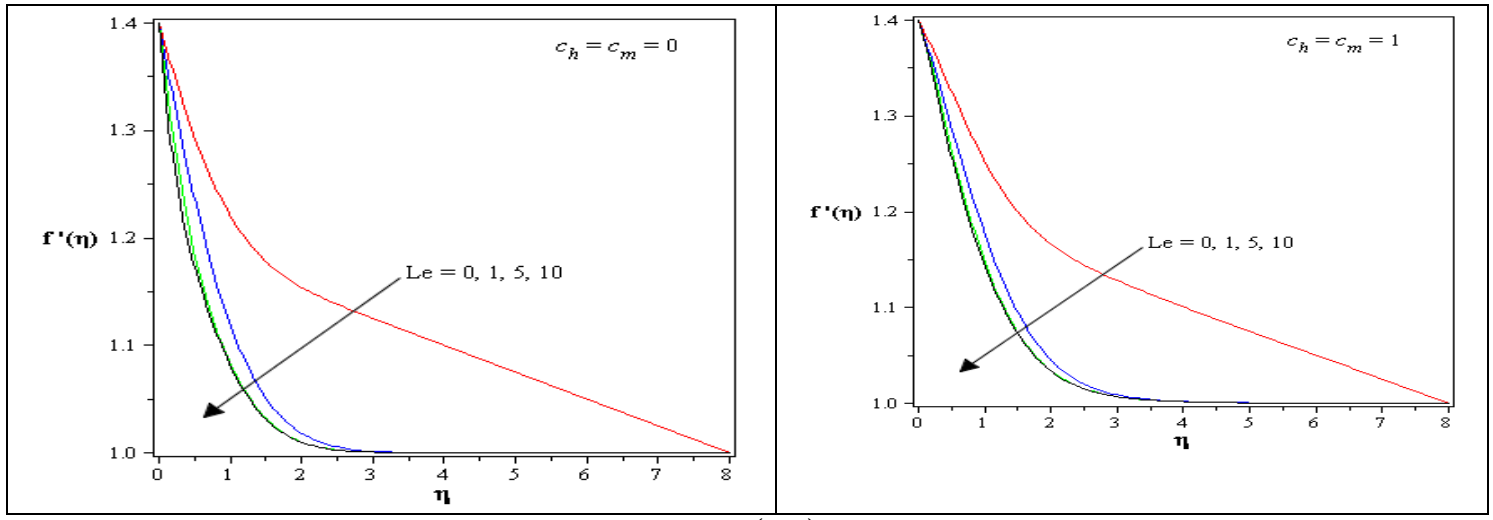

Fig.2: Effects of Lewis number $(L e)$ on velocity profiles when

$M=1, N=1, m=1, D_{f}=0.15, S_{r}=0.4, \varepsilon=0.4, \lambda=1 / 4$ 

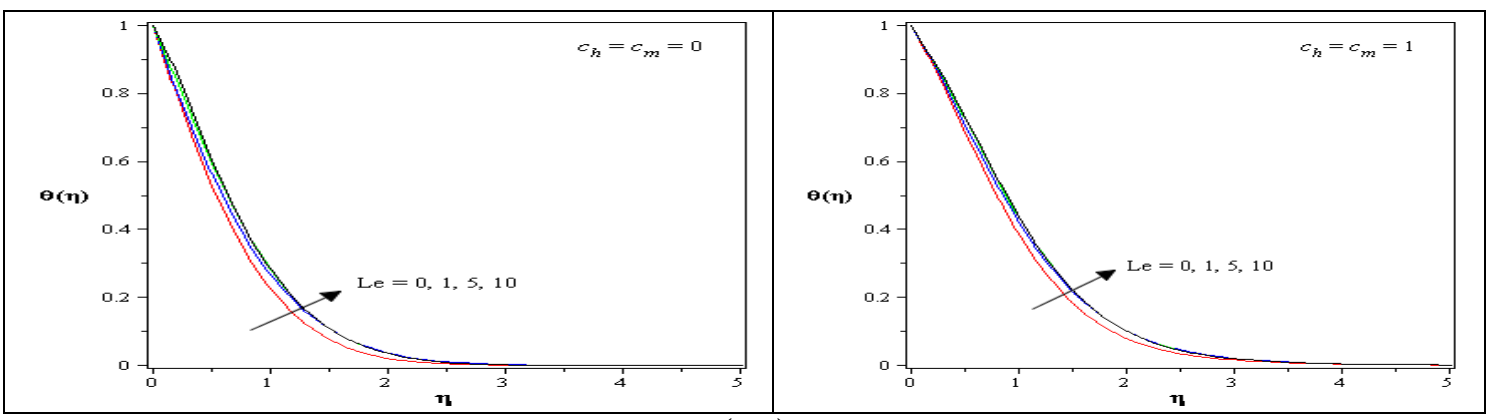

Fig.3: Effects of Lewis number $(L e)$ on temperature profiles when $M=1, N=1, m=1, D_{f}=0.15, S_{r}=0.4, \varepsilon=0.4, \lambda=1 / 4$

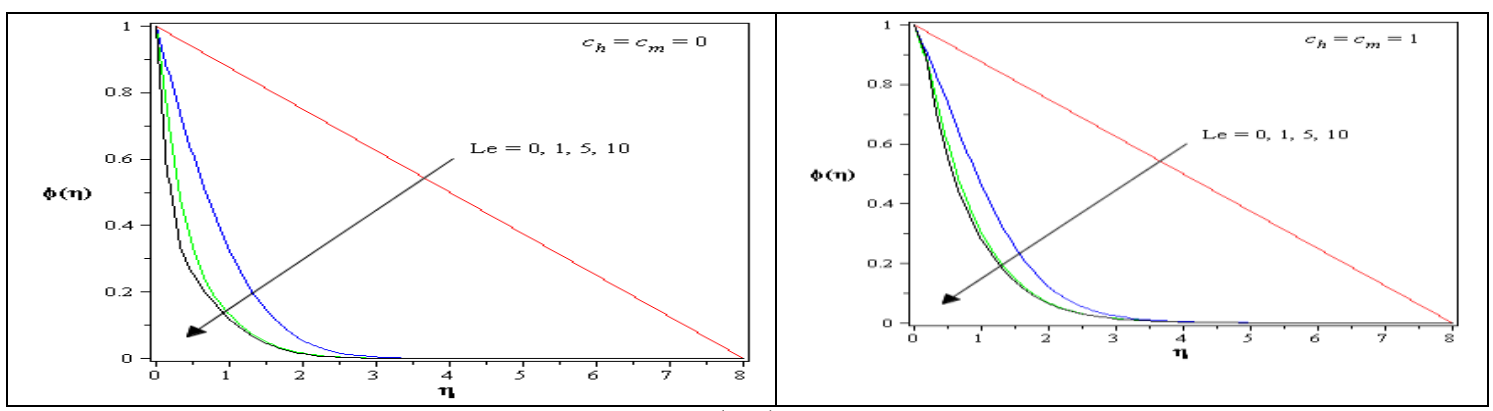

Fig.4: Effects of Lewis number $(L e)$ on concentration profiles when

$M=1, N=1, m=1, D_{f}=0.15, S_{r}=0.4, \varepsilon=0.4, \lambda=1 / 4$

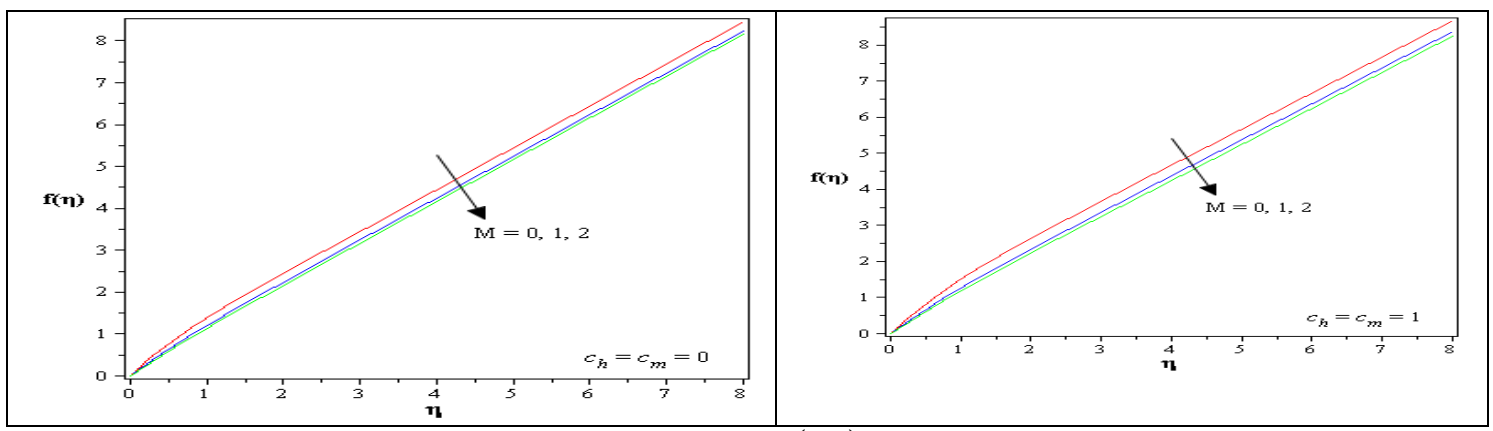

Fig.5: Effects of Magnetic parameter $(M)$ on distance profiles when

$L e=5, N=1, m=1, D_{f}=0.15, S_{r}=0.4, \varepsilon=0.4, \lambda=1 / 4$

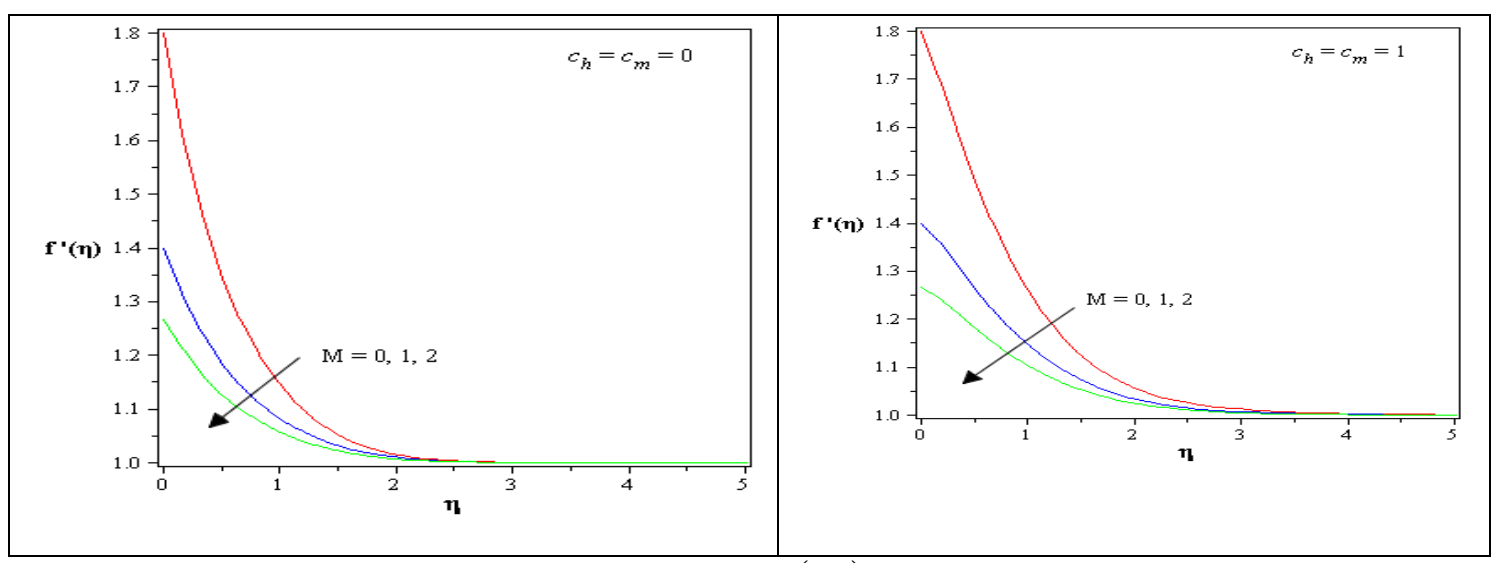

Fig.6: Effects of Magnetic parameter $(M)$ on velocity profiles when $L e=5, N=1, m=1, D_{f}=0.15, S_{r}=0.4, \varepsilon=0.4, \lambda=1 / 4$ 


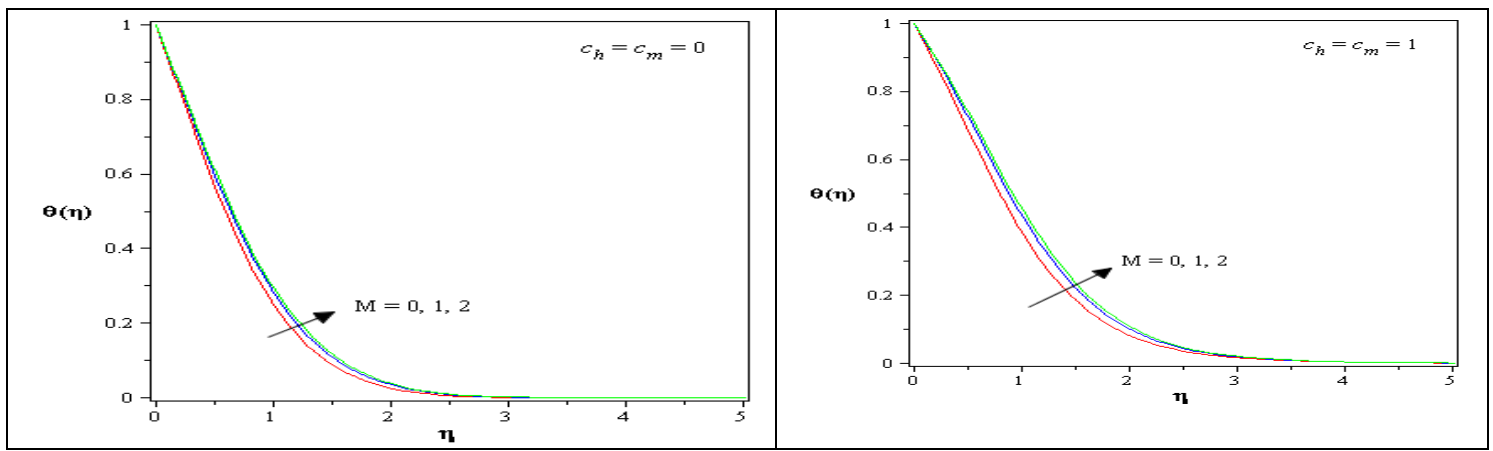

Fig.7: Effects of Magnetic parameter $(M)$ on temperature profiles when $L e=5, N=1, m=1, D_{f}=0.15, S_{r}=0.4, \varepsilon=0.4, \lambda=1 / 4$

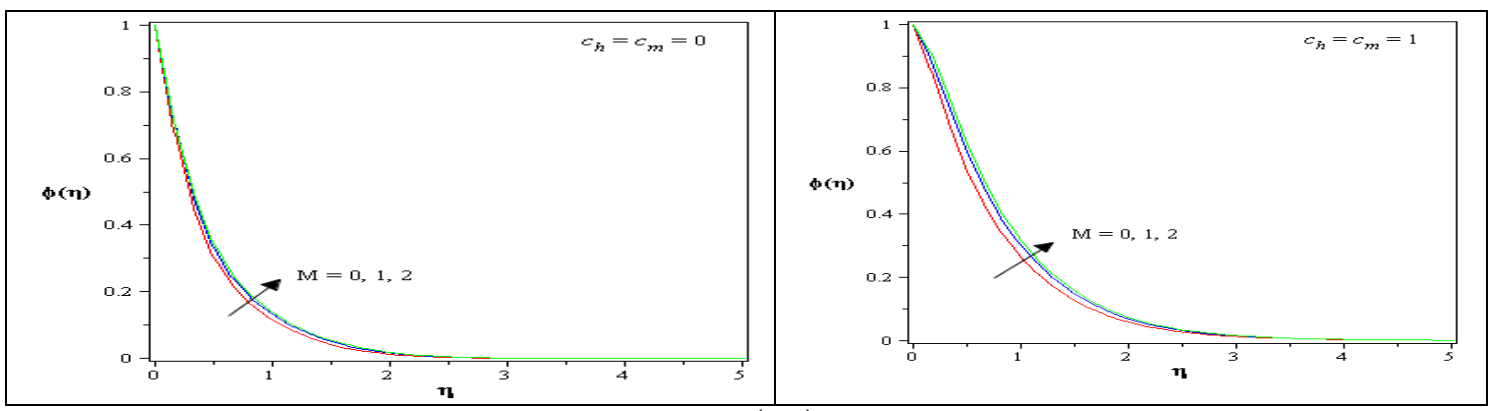

Fig.8: Effects of Magnetic parameter $(M)$ on concentration profiles when

$L e=5, N=1, m=1, D_{f}=0.15, S_{r}=0.4, \varepsilon=0.4, \lambda=1 / 4$

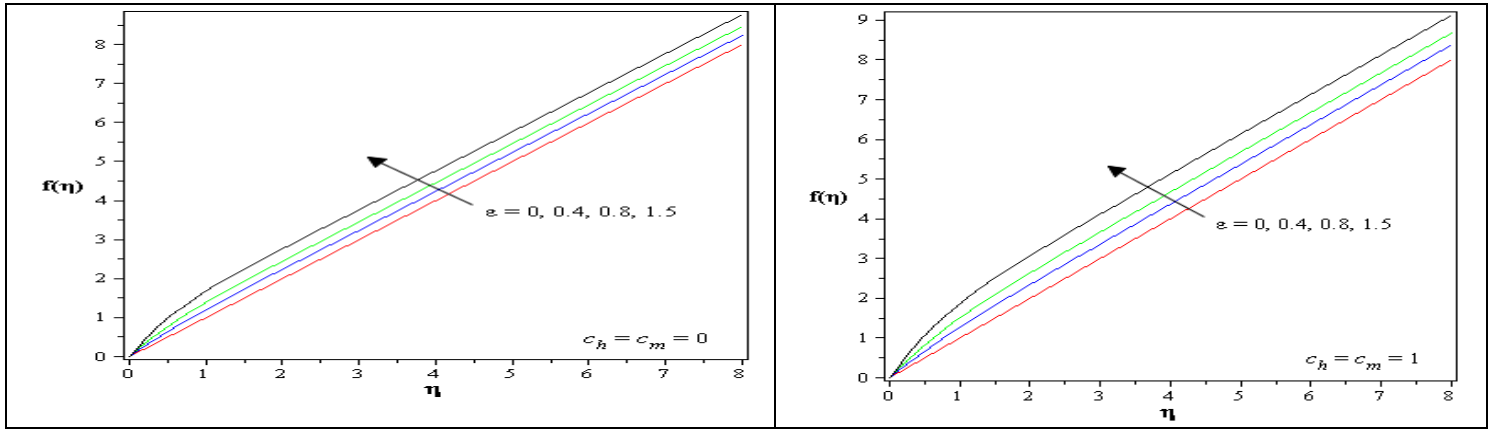

Fig.9: Effects of Mixed convection parameter $(\varepsilon)$ on distance profiles when

$$
L e=5, N=1, m=1, D_{f}=0.15, S_{r}=0.4, M=1, \lambda=1 / 4
$$
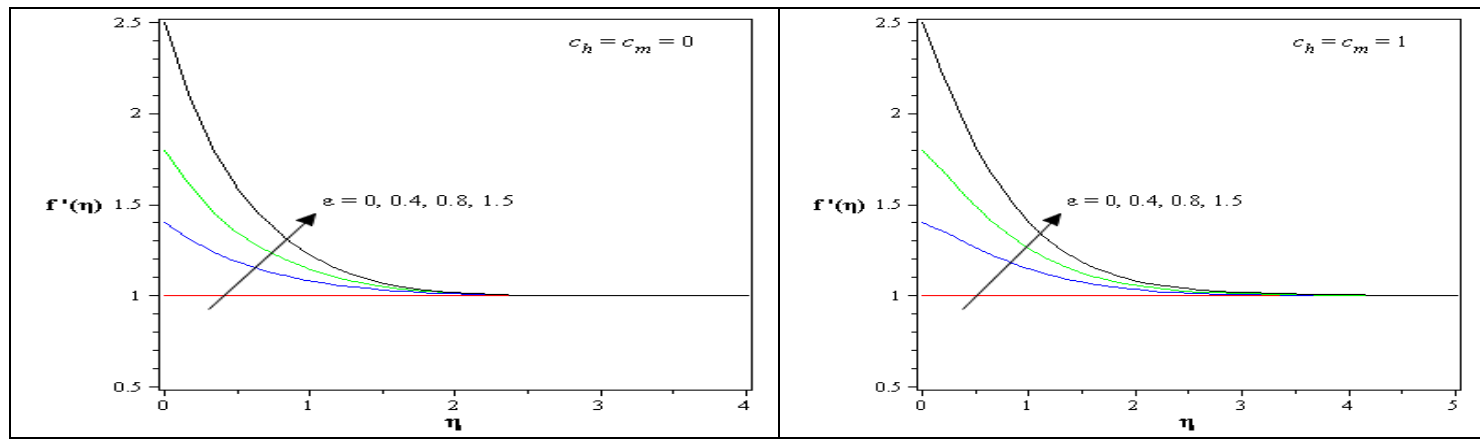

Fig. 10: Effects of Mixed convection parameter $(\varepsilon)$ on velocity profiles when

$$
L e=5, N=1, m=1, D_{f}=0.15, S_{r}=0.4, M=1, \lambda=1 / 4
$$



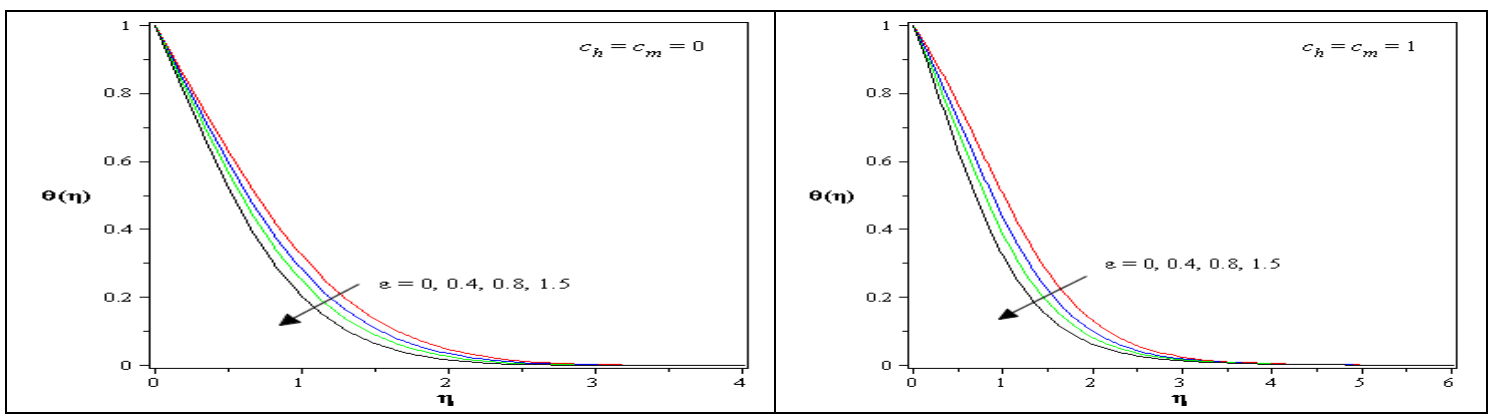

Fig. 11: Effects of Mixed convection parameter $(\varepsilon)$ on temperature profiles when

$$
L e=5, N=1, m=1, D_{f}=0.15, S_{r}=0.4, M=1, \lambda=1 / 4
$$

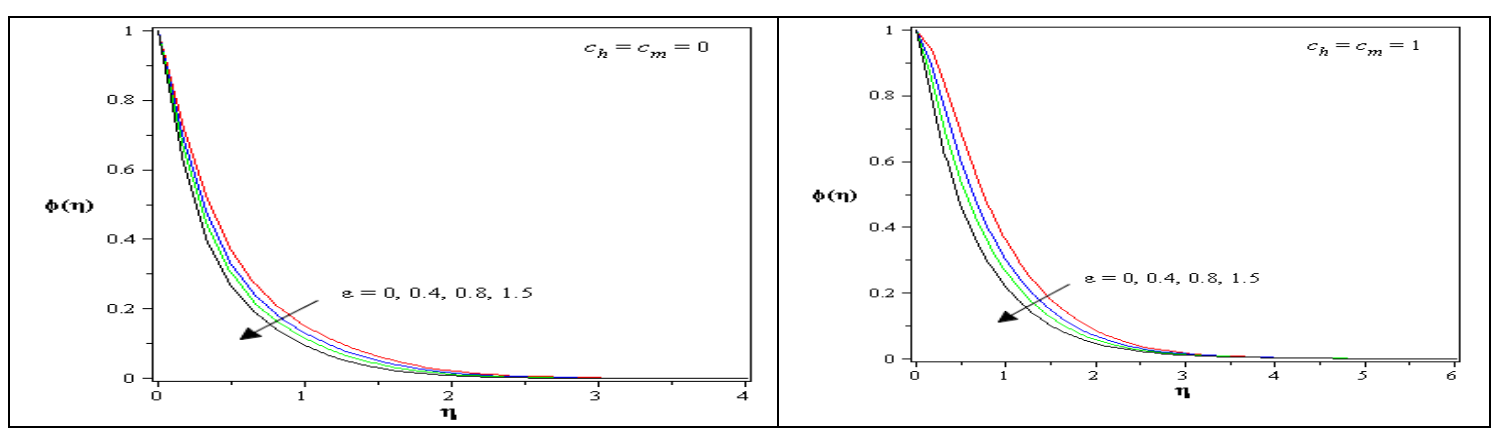

Fig. 12: Effects of Mixed convection parameter $(\varepsilon)$ on concentration profiles when

$$
L e=5, N=1, m=1, D_{f}=0.15, S_{r}=0.4, M=1, \lambda=1 / 4
$$

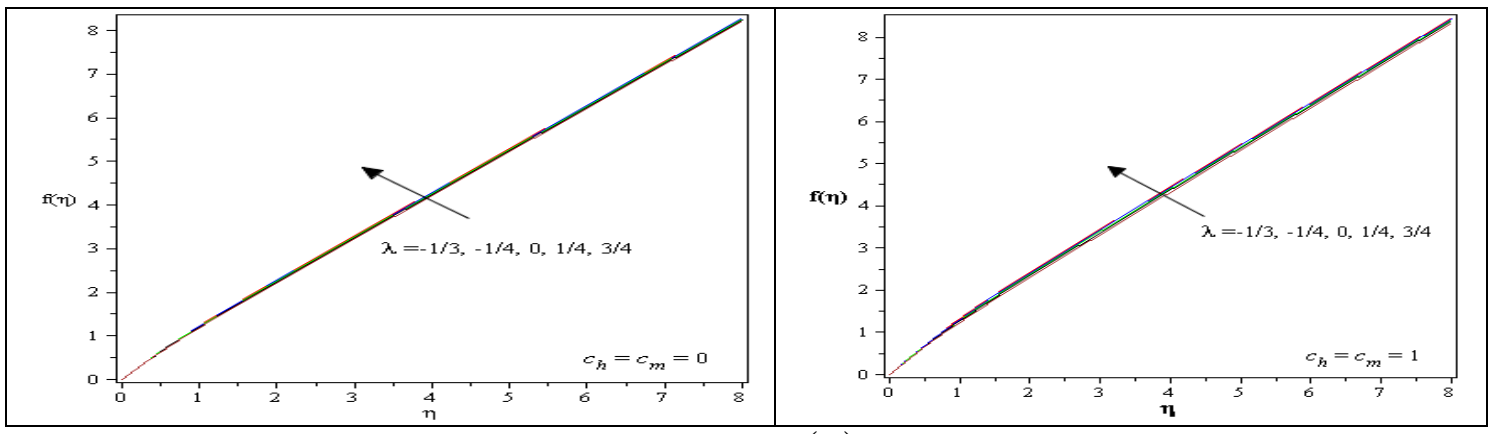

Fig. 13: Effects of exponent constant $(\lambda)$ on distance profiles when

$L e=5, N=1, m=1, D_{f}=0.15, S_{r}=0.4, M=1, \varepsilon=0.4$
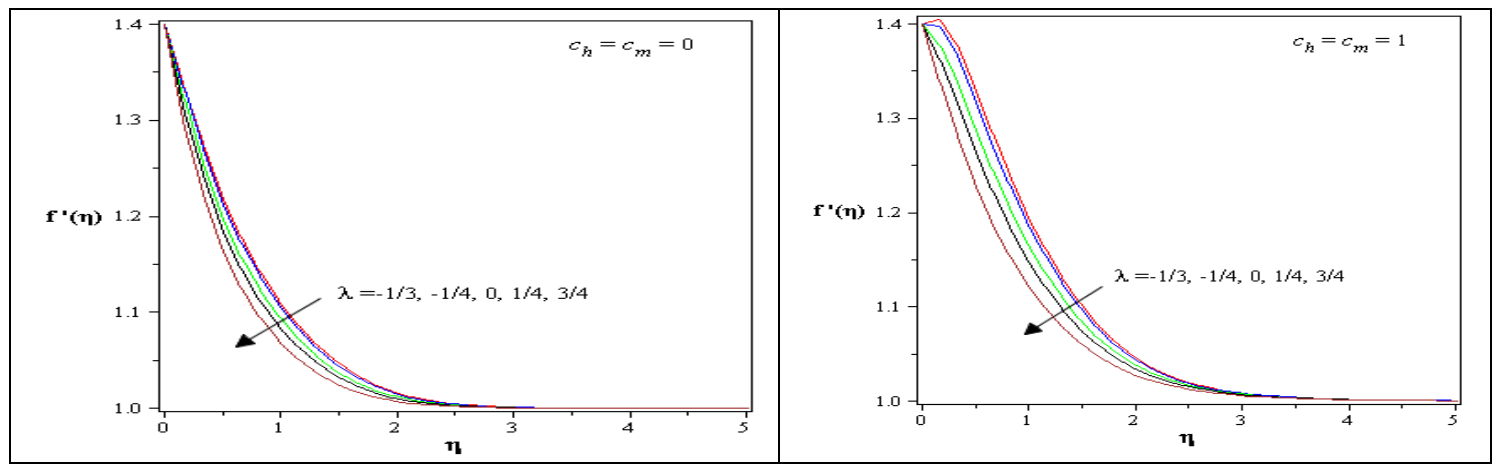

Fig. 14: Effects of exponent constant $(\lambda)$ on velocity profiles when

$L e=5, N=1, m=1, D_{f}=0.15, S_{r}=0.4, M=1, \varepsilon=0.4$ 

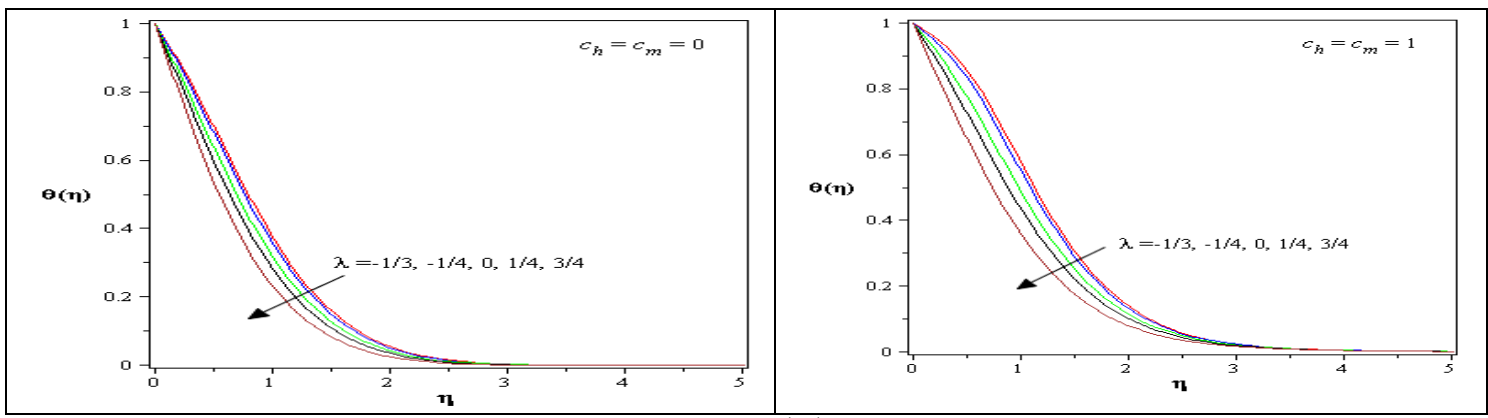

Fig. 15: Effects of exponent constant $(\lambda)$ on temperature profiles when

$L e=5, N=1, m=1, D_{f}=0.15, S_{r}=0.4, M=1, \varepsilon=0.4$
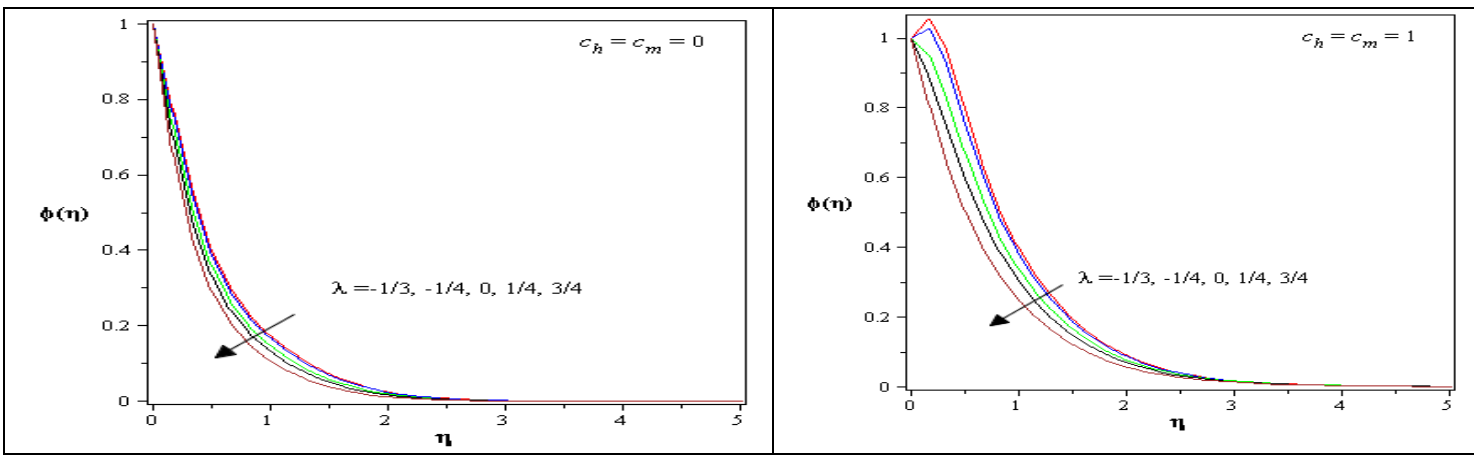

Fig. 16: Effects of exponent constant $(\lambda)$ on concentration profiles when

$$
L e=5, N=1, m=1, D_{f}=0.15, S_{r}=0.4, M=1, \varepsilon=0.4
$$
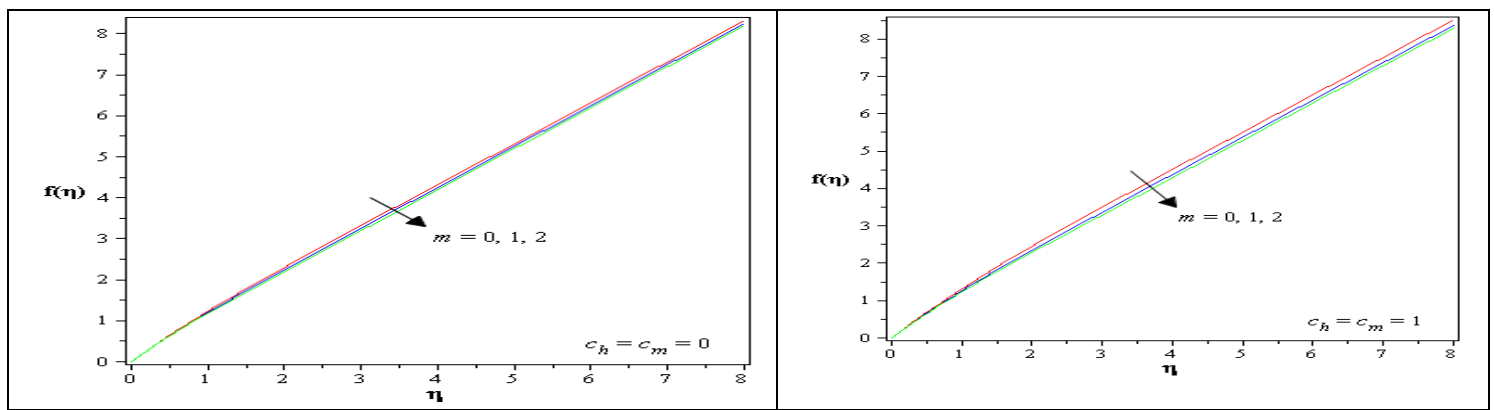

Fig. 17: Effects of $m$ on distance profiles when

$$
L e=5, N=1, m=1, D_{f}=0.15, S_{r}=0.4, M=1, \varepsilon=0.4
$$

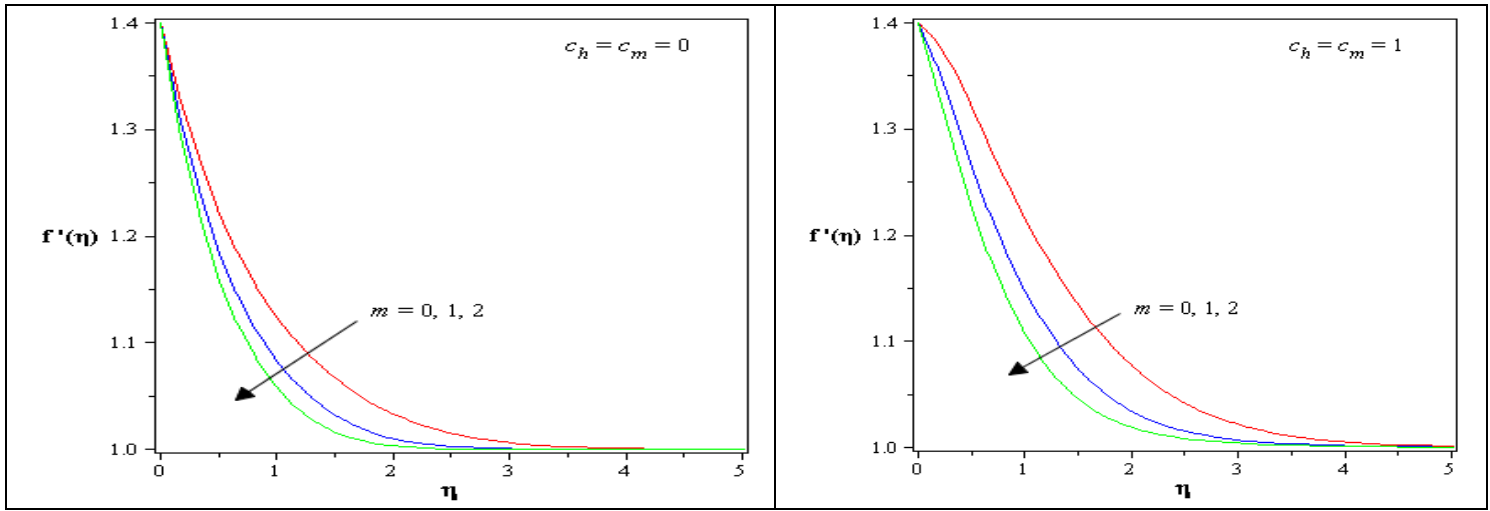

Fig. 18: Effects of $m$ on velocity profiles when

$L e=5, N=1, m=1, D_{f}=0.15, S_{r}=0.4, M=1, \varepsilon=0.4$ 


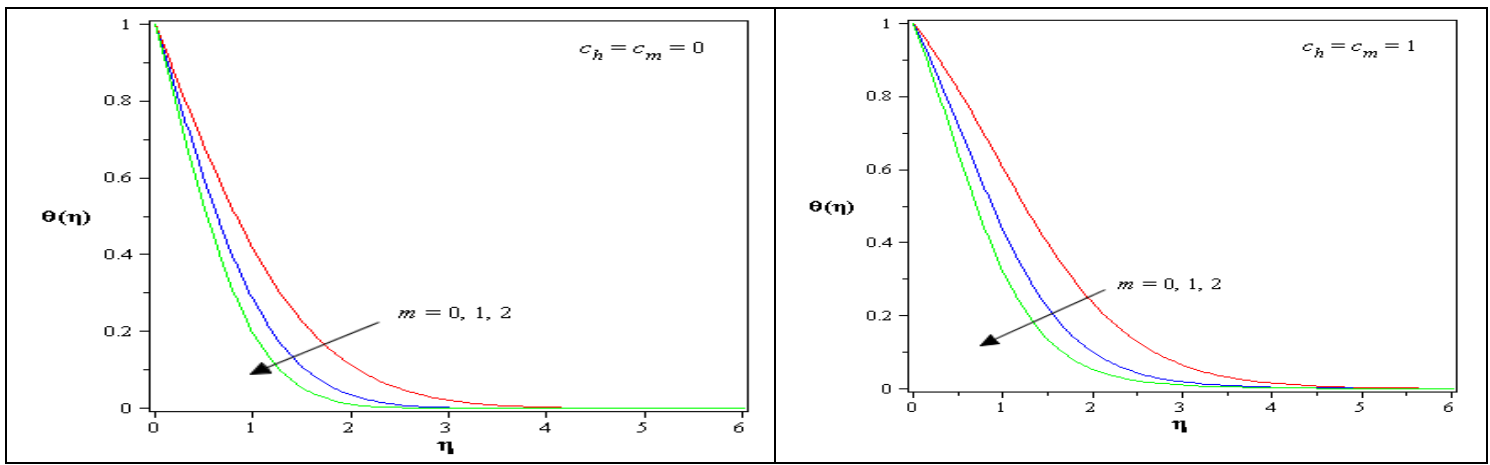

Fig. 19: Effects of $m$ on temperature profiles when

$L e=5, N=1, m=1, D_{f}=0.15, S_{r}=0.4, M=1, \varepsilon=0.4$
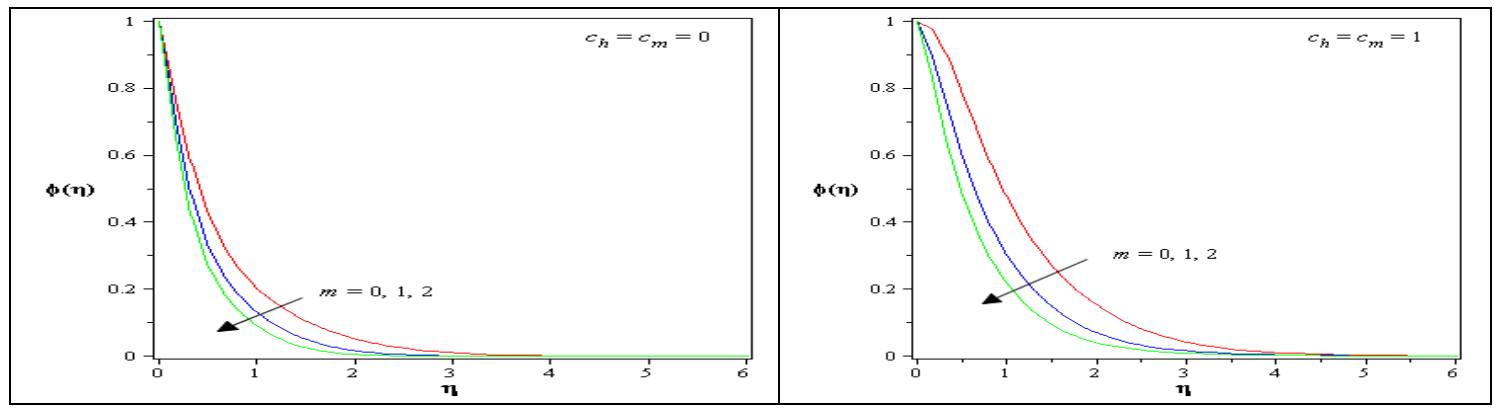

Fig. 20: Effects of $m$ on concentration profiles when

$L e=5, N=1, m=1, D_{f}=0.15, S_{r}=0.4, M=1, \varepsilon=0.4$

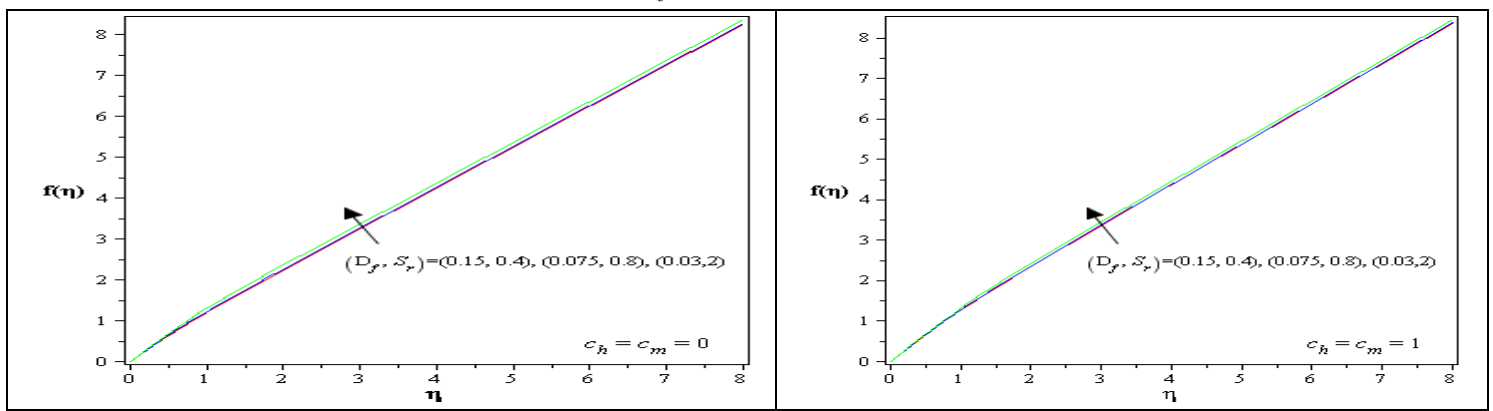

Fig. 21: Effects of $\left(D_{f}, S_{r}\right)$ on distance profiles when

$L e=5, N=1, m=1, \varepsilon=0.4, M=1, \lambda=1 / 4$

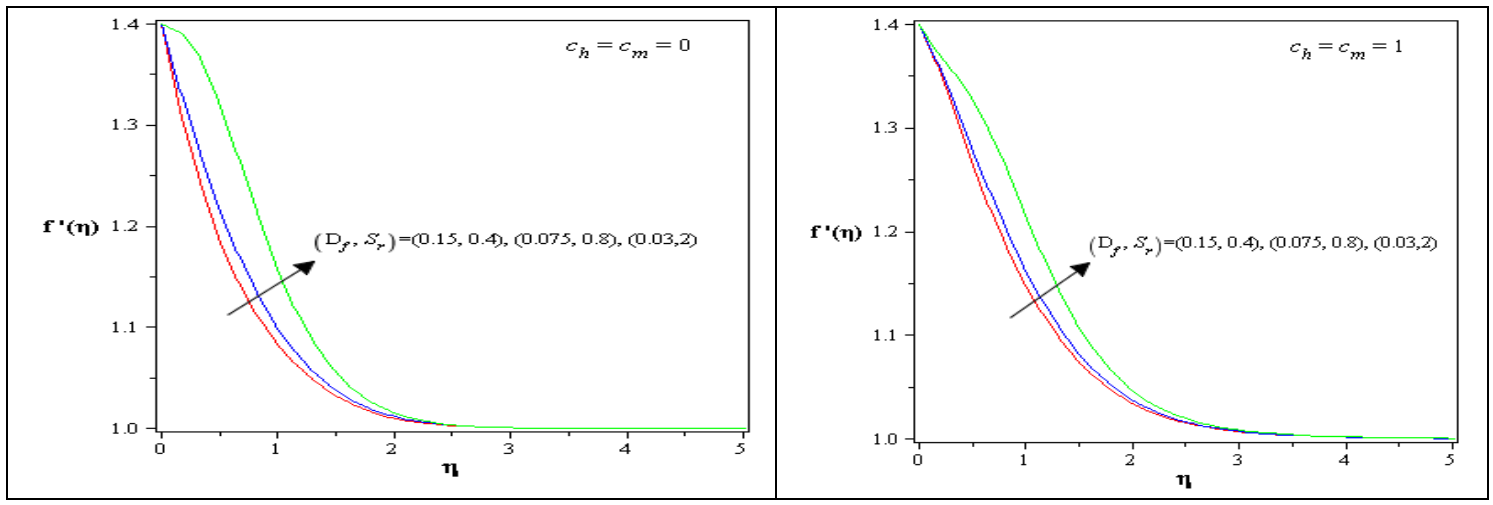

Fig. 22: Effects of $\left(D_{f}, S_{r}\right)$ on velocity profiles when

$L e=5, N=1, m=1, \varepsilon=0.4, M=1, \lambda=1 / 4$ 

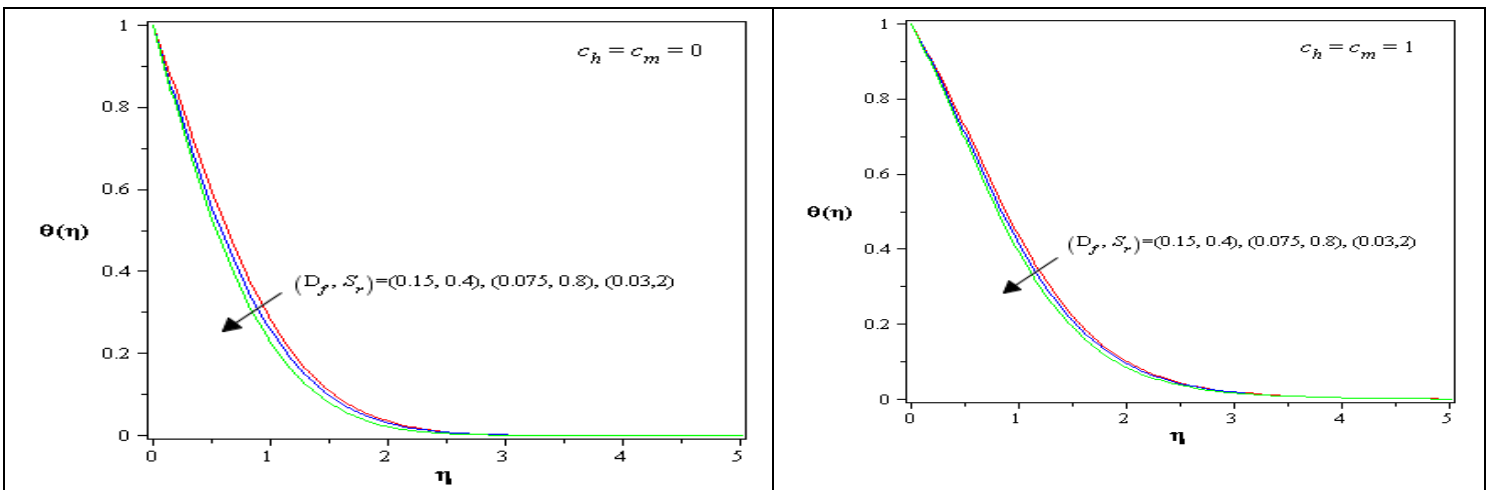

Fig. 23: Effects of $\left(D_{f}, S_{r}\right)$ on temperature profiles when

$L e=5, N=1, m=1, \varepsilon=0.4, M=1, \lambda=1 / 4$

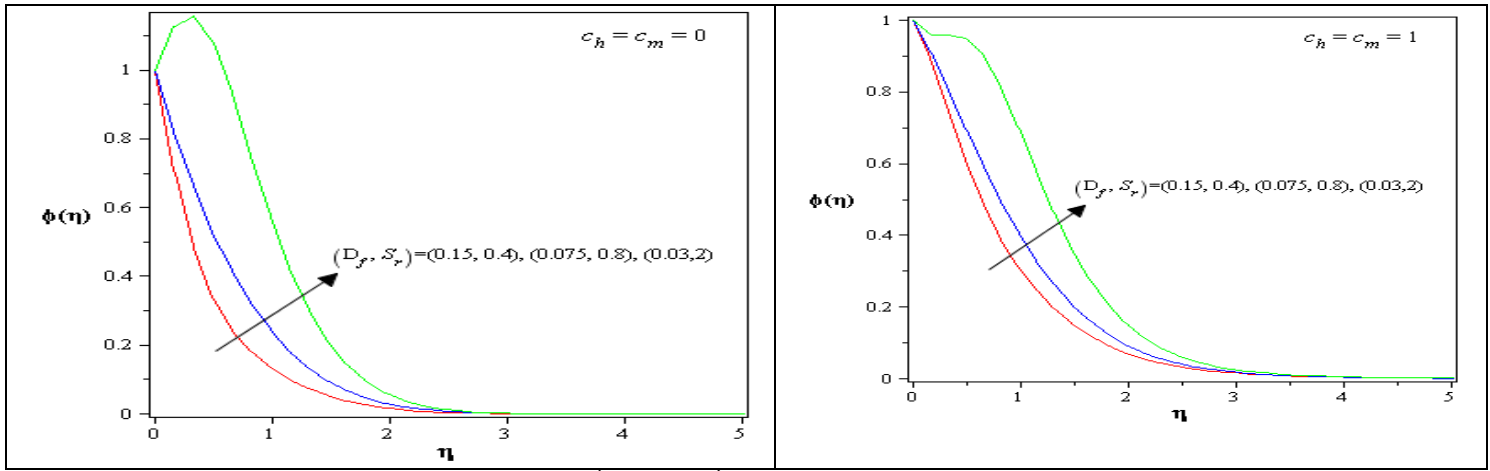

Fig. 24: Effects of $\left(D_{f}, S_{r}\right)$ on concentration profiles when

$$
L e=5, N=1, m=1, \varepsilon=0.4, M=1, \lambda=1 / 4
$$

\section{Conclusion}

In this study an analysis has been done to investigate the effects of mixed convection parameter $\varepsilon$, magnetic parameter $M$, exponent constant $\lambda$, Lewis number $L e$, Soret number $S_{r}$ and Dufour number $D_{f}$ on heat and mass transfer over a steady MHD free convection flow over a vertical surface in porous media in the presence of internal heat generation. It has been found that these parameters affect considerably the considered flow characteristics.

\section{References}

[1]. Tanner, R.I. 1992. Engineering Rheology. Oxford University Press: Oxford, UK

[2]. Eckeret ERG and Drake RM (1972). Analysis of heat and mass transfer. McGraw Hill, New York.

[3]. Dursunkaya Z and Worek WM (1992). Diffusion-thermo and thermal diffusion effects in transient and steady natural convection from a vertical surface. International Journal of Heat and Mass Transfer 35, pp. 2060-2065.

[4]. Kafoussias NG and Williams NG (1995). Thermal-diffusion and diffusion-thermo effects on mixed free-forced convective and mass transfer boundary layer flow with temperature dependent viscosity. International Journal of Engineering Science 33, pp. 13691384.

[5]. Postelnicu A (2004). Influence of a magnetic field on heat and mass transfer by natural convection from vertical sufaces in porous media considering Soret and Dufour effects. International Journal of Heat and Mass Transfer 47, pp. 1467-1475.

[6]. Abreu CRA, Alfradique MF, and Silva AT (2006). Boundary layer flows with Dufour and Soret effects: I: Forced and natural convection. Chemical Engineering Science 61, pp. 4282-4289.

[7]. Lakshmi Narayana PA and Murthy PVSN (2007). Soret and Dufour effects in a doubly stratified Darcy porous medium. Journal of Porous Media 10, pp. 613-624.

[8]. Eringen AC (1966). Theory of micropolar fluids. Journal of Mathematics and Mechanics 16, pp. 1-18.

[9]. Lukaszewicz G (1999). Micropolar fluids - Theory and Applications. Birkhauser, Basel. Markin JH and Mahmood T (1989). Mixed convection boundary layer similarity solution, prescribed heat flux. Zeitschrift fuer angewandte Mathematik und Physik 40, pp. 6168.

[10]. Ahmadi G (1976). Self-similar solution of incompressible micropolar boundary layer flow over a semi-infinite plate. International Journal of Engineering Science 14, pp. 639-646.

[11]. Jena SK and Mathur MN (1984). Mixed convection flow of a micropolar fluid from an isothermal vertical plate. Computers \& Mathematics with Applications 10, pp. 291-304.

[12]. Gorla RSR (1992). Mixed convection in a micropolar fluid from a vertical surface with uniform heat flux. International Journal of Engineering Science 30, pp. 349-358. 
[13]. Rahman MM, Eltayeb IA, and Rahman SMM (2009). Thermo-micropolar fluid flow along a vertical permeable plate with uniform surface heat flux in the presence of heat generation. Thermal Science 13, pp. 23-36.

[14]. Beg OA, Bhargava R, Rawat S, and Kahya E (2008). Numerical study of micropolar convective heat and mass transfer in a nonDarcy porous regime with Soret and Dufour effects. Emirates Journal for engineering research 13, pp. 51-66.

[15]. Rawat S and Bhargava R (2009). Finite element study of natural convection heat and mass transfer in a micropolar fluid saturated porous regime with Soret/Dufour effects. International Journal of Applied Mathematics and Mechanics 5, pp. 58-71.

[16]. Gnaneswara Reddy M and Bhaskar Reddy N (2010). Soret and dufour effects on steady MHD free convection flow past a semiinfinite moving vertical plate in a porous medium with viscous dissipation. Int. J. of Appl. Math and Mech 6 (1), pp. 1-12.

[17]. Ghosh SK, Bg OA, Bhargava R, Rawat S and Bg TA (2010). Mathematical modelling of transient magnetohydrodynamic couple stress fluid flow in a rotating channel. Int. J. of Appl. Math and Mech 6(6), pp. 23-45.

[18]. Ramana Murthy JV, Muthu P and Nagaraju G (2010). Finite difference solution for mhd flow of couple stress fluid between two concentric rotating cylinders with porous lining. Int. J. of Appl. Math and Mech. 6 (12), pp. 1-28. 